\title{
Effect of resistance exercise training on Crohn's disease patients
}

\author{
Konstantinos Papadimitriou \\ Laboratory of Evaluation of Human Biological Performance, School of Physical Education and Sport Science, Aristotle University of \\ Thessaloniki, Thessaloniki, Greece
}

Crohn's disease (CD) is observed with increased levels of cytokines which cause inflammation in many parts of the digestive tract. Aerobic exercise contributes to the reduction of the intestine's inflammation and increases the quality of life. Another type of exercise that shows research interest about its effects on CD symptoms is the resistance exercise (RE). The aim of the study was to review the influence of RE on CD patients. The study's literature was collected from PubMed and Scholar databases. According to the results, the main phase of a RE training program must have a gradual increase of intensity $(60 \%-80 \%)$ and resting periods of 15-30 seconds after each exercise, and 2-3 minutes between exercises. Also, CD patients who were in remission improved the muscle strength and quality of life via their participation in RE training program. However, the secretion of interleukin-6 in both CD and RE contributes in various physiological mechanisms setting a contradictory role in the effectiveness of $\mathrm{RE}$ at the disease's inflammatory situation. So, the use of RE training in CD patients needs more research for safer participation. (Intest Res 2021;19:275-281)

Key Words: Inflammation; Intestine; Cytokines

\section{INTRODUCTION}

According to the literature, a parameter that enhances the antiinflammatory action in Crohn's disease (CD) patients is physical exercise. American Academy of Sports Medicine recommends either moderate-intensity aerobic exercise for a minimum of 30 minutes 5 days each week or vigorous-intensity physical activity for 20 minutes 3 days a week. However, there is only one published set of guidelines for exercise in patients with inflammatory bowel disease (IBD). ${ }^{1}$

Many studies present the beneficial role in different types of exercise. Mainly, the aerobic type of exercise has been found of being used in many cases from CD patients, especially in

Received April 29, 2020. Revised September 4, 2020.

Accepted September 7, 2020.

Correspondence to Konstantinos Papadimitriou, School of Physical

Education and Sport Science, Aristotle University of Thessaloniki, Thetidos

5, Thessaloniki 54636, Greece. Tel: +30-6980265800, Fax: +30-2221022973,

E-mail: kostakispapadim@gmail.com remission. The aerobic exercise contributes through its antiinflammatory activity, ${ }^{2-6}$ the reduction of apoptotic protein expression $^{6,7}$ and the maintenance of musculature. ${ }^{6.9}$ Nevertheless, anti-inflammatory activity against IBD such as CD has not yet been described precisely. ${ }^{2}$

Legeret et al. ${ }^{2}$ describes the anti-inflammatory mechanism through the release of myokines from the skeletal muscles, such as interleukins (IL- $6^{2,10}$ and IL-15). ${ }^{6}$ IL-6 regulates the production of anti-inflammatory factors such as IL-1 antagonist and IL-10. ${ }^{11}$ Furthermore, it inhibits the pro-inflammatory cytokine tumor necrosis factor- $\alpha$ (TNF- $\alpha$ ). Marchioni Beery et al. $^{12}$ reported adrenaline as another possible factor that inhibits the production of TNF- $\alpha$. IL-15 is described as an anti-inflammatory factor, too, which is expressed in human skeletal muscle and has been identified as an anabolic factor in muscle growth. ${ }^{13}$ Glucagon-like peptides and Irisin increase through the exercise too. Both, contribute to trophic growth factors that enhance and repair the damaged intestinal mucosa. ${ }^{13,14}$ 
Moreover, the exercise's anti-inflammatory activity shows an association with the heat shock proteins (HSP), which are known as proteins that down-regulate the secretion of pro-inflammatory cytokines. ${ }^{2,10}$ In Chen and Noble ${ }^{10}$ review is reported that isotonic contractions, such as these which are associated with endurance-type activities, tend to lead to increases in HSP 60 and 70. In the eccentric type of exercise, increases are observed in phosphorylation, and translocation of HSP 25/27 and in aB-crystallin. Generally, these secretions of HSP contribute to the protection of many tissues such as mitochondria, sarcoplasmic reticulum, and cytoskeletal protection. ${ }^{10}$

On the other hand, it has been observed that forced exercise may exacerbate inflammation. ${ }^{13}$ It is interesting that intensive exercise in humans, such as long-distance running, could lead to "runner's ischemic colitis" involving bloody diarrhea, fatigue, and fever. ${ }^{3,4}$ In CD patients found that maximum exercise load was limited in adults. ${ }^{15}$ Long term, high-intensity exercise may induce mild systemic inflammation and an increased level of cytokines in both active and remission CD patients. These adverse effects depend on the intensity and duration of exercise. $^{13}$

Another type of exercise which CD patients use is resistance exercise (RE). However, the physiology mechanism of RE in CD patients is not quite clear if it enhances the anti-inflammatory action or involves an exacerbation of the disease's symptoms. So, the aim of the study was to examine the effect of RE on CD patients' physiology and health.

\section{METHODS}

The data were collected from PubMed, MEDLINE, and Google Scholar databases to identify the effect of exercise in CD, and the effect of RE on CD patients. These databases were chosen because of the including studies in the fields of health and exercise. The combinations of terms that used were: "Crohn," "inflammation," "intestine," "bowel," "disease" with both "exercise" and "resistance training." The articles were chosen through some inclusive and exclusive criteria. The inclusive criteria were: (1) the articles must be in the English language, (2) CD studies in humans and animals, (3) the effect of aerobic exercise through an experimental process, and (4) the effect of RE through an experimental process. On the other hand, the exclusion criteria were: (1) not the inclusion criteria and (2) studies that used only questionnaires in their methodology. For the manuscript, 39 studies were used. Specifically, 5 were for CD inflammatory mechanisms in different populations, 2 for
$\mathrm{CD}$ in animals, 16 for the effect of exercise in $\mathrm{CD}, 8$ for the $\mathrm{RE}$ and the mechanism of muscle hypertrophy, and 8 studies for the effect of RE on CD patients.

\section{RESULTS}

RE enhances the adaptive response of the skeletal muscles to induce hypertrophy via increasing muscle mass. ${ }^{16}$ Muscle hypertrophy is induced through the increasing of mechanical tension, metabolic stress, and muscle damages which activate the inflammation response through muscle cells' swelling. ${ }^{17}$ The pro-inflammatory cytokines which were observed through the inflammation are IL- $1 \beta$, TNF- $\alpha$, while the anti-inflammatory cytokines are IL-1 receptor antagonist, IL-1RA, IL-10, and pleiotropic IL-6. ${ }^{18,19}$

Both regular concentric and isometric RE, instead of eccentric exercise which induces greater muscle damage and inflammation, are beneficial to upregulating defense mechanisms against oxidative stress. ${ }^{20}$ Also, through phosphatidylinositol3-OH kinase-Akt (PI3K-Akt)/mammalian target of rapamycin (mTOR), mitogen activated protein kinase (MAPK), and $\mathrm{Ca}^{2+}$ pathways induce protein synthesis and muscle growth. ${ }^{21}$ Moreover, the hormonic anabolic process, such as hepatocyte growth factor and myokines as IL-5, and IL-6, ${ }^{18} \mathrm{IL}-10,{ }^{1}$ fibroblast growth, and leukemia inhibitory factor, promotes the anabolism and anti-inflammation. ${ }^{17}$

Wiroth et al. ${ }^{22}$ and Cabalzar et al. ${ }^{23}$ highlighted that CD patients in both remission and active phase showed decrements on the strength performance such as limb strength, limb endurance, and sit-up test concerning healthy participants. ${ }^{22}$ Also, showed a lower respiratory and peripheral muscle strength. ${ }^{23}$ Thus, in both CD conditions, RE training seems to be an important factor that probably enhances patients' muscle performance and quality of life.

One of the first studies which examined the possible effect of RE in CD patients with low symptoms activity, was conducted by Robinson et al. ${ }^{24}$ They examined the effect of RE on bone mineral density. The program involved a 5 -minute warm-up for the whole body and a 5-minute rest after the warm-up. The program's main body involved 12 core floorbased low-impact exercises which were focusing on the major muscle groups of the trunk and legs.

The program was conducted in 10 sessions per month and the intensity of exercises was increased by developing the core exercises, increasing the number of repetitions of each exercise, advancing body positions to increase resistance to 
movement, and using resistive tubing or free weights. According to the results, there was a positive effect on bone mineral density and no exacerbation activity of the symptoms. ${ }^{24}$

The first randomized controlled trial which examined the effect of RE training program in patients with IBD was conducted by Candow et al. ${ }^{25}$ After a three-time per week program with 12 REs at $60 \%$ to $70 \%$ of 1-repetition maximum (RM), improvement observed in muscle strength. Then, Pérez ${ }^{26}$ tried to guide the structure of an ideal RE training program for CD patients.

Thus, they proposed that an RE training program should start with a 5-minute warm-up and whole-body mobility. Then, as a main part of the training session, 2 sets of 10-12 muscular REs should focus on the major muscle groups of the trunk and legs. Moreover, in REs, elastic bands and free weights could be utilized. In both types of REs, the patients should gradually adjust the intensity increasing the repetitions or the load, respectively. ${ }^{26}$

In an 8-week RE program which was used in CD patients by de Souza Tajiri et al., ${ }^{27}$ the quadriceps strength was examined. In the first 4 weeks of the program, the participants trained 3 sets of 12 repetitions at $50 \%$ of the maximum load increasing gradually the load until $80 \%$ in the final week. At the end of the 8-week program, the participants had been improved in maximal isometric quadriceps strength and in quadriceps 1-RM.

Cronin et al. ${ }^{28}$ examined the effect of a combined aerobic and RE training program through an 8-week intervention. The $\mathrm{RE}$ training program included 7 machine-based REs with a minimum required to perform 3 sets of 8 repetitions. The intensity was calculated inductively at $70 \%$ of the individual's 1-RM value. During the intervention, there was a progressive increase in the intensity of exercises from $15 \%$ to $20 \%$.

According to the results, it was indicated that via the combination between aerobic exercise and $\mathrm{RE}$ the $\mathrm{VO}_{2}$ max was improved from $43.41 \mathrm{~mL} / \mathrm{kg} / \mathrm{min}$ to $46.01 \mathrm{~mL} / \mathrm{kg} / \mathrm{min}$, the body fat decreased at $2.1 \%$ while the lean tissue mass increased by $1.59 \mathrm{~kg}$. Also, the pro-inflammatory cytokines such as IL-8, IL10, IL-6, and TNF- $\alpha$, and circulating levels of C-reactive protein were stable at the begging of the intervention and probably were not influenced during the intervention. ${ }^{28}$

Through the muscle hypertrophy mechanism, the RE induces inflammation in muscles via the damaging of the tissue. ${ }^{17,29}$ So, there is a guideline in which CD patients must use $\mathrm{RE}$ at low to moderate intensity in order to avoid possible disease exacerbation. ${ }^{13}$ Moreover, the patients must avoid fatigue, thus, it is preferable to rest almost for 15-30 seconds after each exercise and for 2-3 minutes between sets. ${ }^{26}$

\section{DISCUSSION}

$\mathrm{CD}$ is the intestine's increasing inflammatory situation. This inflammation occurring by cytokines such as TNF- $\alpha$, and ILs. ${ }^{30,31}$ According to the literature, the effect of low intensity aerobic and RE induces an anti-inflammatory mechanism against TNF- $\alpha$ and some ILs which cause the intestine inflammation. ${ }^{13,14,27}$ However, the lack of literature about the effects of RE on CD patients makes further research necessary.

CD patients' participation in RE training program in both remission and active situations seems to be necessary for the enhancement of muscle performance. ${ }^{22,23}$ According to the 4 studies that used RE in CD patients found that the program must be structured ${ }^{26}$ and include a warm-up period, a main phase with exercises with a gradual increase of the intensity between $60 \%$ and $80 \%$ of 1-RM and resting periods of $15-30$ seconds after each exercise and 2-3 minutes between sets. The number of exercises varies from 7 to 12 and involves bodyweight exercises, machines, resistive tubing, and free weights. Also, the program must occur 2 to 3 times per week and the duration for an effective adaptation is from 8 to 12 weeks. ${ }^{24-28}$

In all studies, the participants were in remission phase and according to the results they improved their muscle strength and quality of life. Also, in intervention studies no exacerbation of symptoms was observed. Whereas, the secretions of pro and anti-inflammatory cytokines were regulated. ${ }^{24-28}$ However, in all studies, there were not any patients in active disease's phase, probably because there is a high risk of the exacerbation of the symptoms during an RE training program. Also, the exercises intensity was low and extended discussion about the physiological mechanism of RE training programs was not observed.

According to the literature, muscle mass hypertrophy through an RE training program, because of the increasing mechanical tension, causes metabolic stress, and muscle damages which activate the inflammation response. ${ }^{17}$ Thus, through the muscle hypertrophy process, there are some intramuscular mechanisms which must be studied and which may cause a potential disease exacerbation.

Between CD and RE conditions, some ILs act as a pro and anti-inflammatory factors. ${ }^{32}$ ILs that contribute to both factors during RE training are IL-6 and IL-10. ${ }^{33,34}$ IL-6 is a cytokine that utilizes in many intracellular functions. ${ }^{35}$ In CD patients, IL-6, IL-10, TNF- $\alpha$, and IL-1 $\beta$ interfere as principal inflammatory cytokines in intestinal epithelia. ${ }^{13,11}$ Carrillo et al. ${ }^{32}$ suggested 
that the increasing levels of IL-6 in blood circulation amplify the inflammatory response in CD. On the other hand, other studies present the exercise as upregulation of the peroxisome proliferator receptor- $\gamma$ coactivator 1- $\alpha$ (PGC-1 $\alpha$ ) factor, which releases IL-6. This secretion acts as anti-inflammatory factor, deregulating the inflammatory cytokines such as TNF- $\alpha$ and IL-1 $1{ }^{18,35}$

Pére ${ }^{26}$ pointed out that to patients who suffer from chronic inflammation diseases, the elevated values of IL-6 might represent a defense mechanism against pro-inflammatory actions which were caused by TNF- $\alpha$. At this point, contradictory results are shown about the effect of IL-6 in CD patients in relation to RE. Specifically, the secretion of IL-6 during an RE training program does not show clear evidence about its action as a pro or anti-inflammatory cytokine. ${ }^{32,33}$

Also, the inflammatory response in skeletal muscles through a muscle hypertrophy program ${ }^{17}$ enhances the hypothesis of Pérez ${ }^{26}$ that IL-6 secretion may imply the negative influence of the exercise on CD patients. Cronin et al. ${ }^{28}$ was the unique who studied the pro-inflammatory cytokines IL-8, IL-10, IL-6, and TNF- $\alpha$, and the circulating levels of C-reactive protein. However, these measurements occurred only before the intervention. Thus, there is not a safe statement about the action of the cytokines during the RE training program.

de Souza Tajiri et al. ${ }^{27}$ in a pilot study revealed that after an 8-week program CD patients had been improved in maximal isometric quadriceps strength and in quadriceps 1-RM. According to the results, no muscular lesions or any exacerbation in intestinal symptoms were found. Instead, patients had an improvement in these. Nevertheless, in this pilot study, there is not any evidence about the ILs' action. Therefore, there is still unclear evidence about the effects of RE in CD patients and the secretion of cytokines.

Lee and Jun ${ }^{31}$ established that the secretion of IL-6 depends on the volume and intensity of the RE training. On the other hand, Raines et al. ${ }^{33}$ concluded that the intensity of exercise does not change the secretion levels of IL-6, whereas, Hirose et $\mathrm{al}^{34}$ claimed that IL-10 response may inhibit the inflammation and promoting adaptation to muscle damages. According to these findings, there is evidence that supports the use of RE on CD patients. As opposed to the above statements, TNF- $\alpha$, IL-1 $\beta$, and IL- 6 were observed as the main secreted cytokines in CD. Also, many authors manifest that IL-6 is the main cause of intestine's inflammation at a RE training program. ${ }^{26,36}$

Another contradiction which was observed is the effect of $\mathrm{RE}$ on muscle hypertrophy ${ }^{26}$ and the theory which describes that IL-6 inhibits muscle hypertrophy. ${ }^{31}$ At this point, there is not any clear mechanism that could describe it. Although the $\mathrm{RE}$ is being used as a method for the increase of lean body mass $^{28}$ on CD patients, some aspects of literature support that the presence of the IL-6 induces muscle atrophy ${ }^{37}$ and lower the muscle strength and power. Also, reduces the secretion of insulin-like growth factor-1, which has an important effect on muscle mass and function. ${ }^{38}$ Therefore, this contradiction of the literature makes risky the participation of $\mathrm{CD}$ patients in RE training programs.

Another IL-6 involvement shows that its transcription and plasma concentration increase when the circulation of the blood glycogen is in low values, through adenosine monophosphate kinase (AMPK) and p38 mitogen-activated protein kinases (p38MAPK) mediators. So, IL-6 acts as mediator signal of hepatic glycogenolysis and gluconeogenesis. ${ }^{33}$ Moreover, IL-6 increases when insulin levels are high through

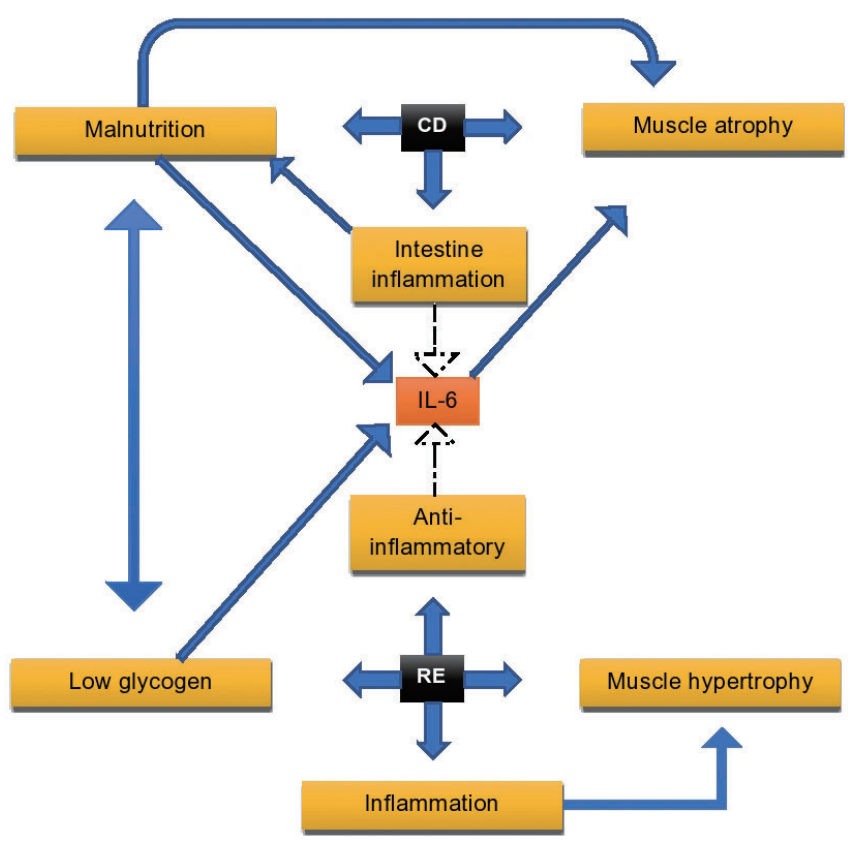

Fig. 1. The physiological connections of interleukin-6 (IL-6) between Crohn's disease (CD) and resistance exercise (RE). IL-6 is being secreted in both CD and RE as inflammatory and anti-inflammatory cytokine respectively. Moreover, the secretion of IL-6 in CD causes malnutrition lowering glycogen's concentrations. After an RE training program, glycogen's concentration lowers too. Intestine's inflammation induces food malabsorption, which causes insufficient energy intake. As a result, the decrease of glycogen concentration in blood circulation induces muscle atrophy. Inhibitor of muscle atrophy is the RE. Despite this, the secretion of IL-6 seems to cause muscle atrophy. Intramuscular inflammation is observed after a RE training program too as a mechanism of muscle hypertrophy. 
AMPK and PI3K-Akt signaling pathways. ${ }^{33}$

Both mechanisms of IL-6 probably connect the inhibition of muscle hypertrophy and the exacerbation of CD after RE. What is more, a frequent symptom in CD is malnutrition which causes muscle atrophy and weakness. Many CD patients in remission demonstrate a decrease in body cell mass compared with healthy persons. Also, loss of muscle mass was found in CD patients as a probable result of food-frequency which is low in fruits, vegetables, milk products, and fish. ${ }^{39}$

Valentini et al. ${ }^{39}$ indicate that the cause of weakness may be observed when the concentration of intramuscular glycogen is in low values. As a result, according to the literature, the IL-6 increases its levels in blood circulation. ${ }^{33}$ In this way, the secretion of IL-6, because of low values of glycogen ${ }^{33}$ in the blood circulation and the RE's energy demands, may manifest an exacerbation of the symptoms in CD.

In the literature, there is a lack of studies about the effect of $\mathrm{RE}$ on CD. However, a common clue in the literature is the effect of the cytokines which mainly contribute as pro and antiinflammatory situations. A cytokine that is repeatedly discussed in many studies about its multifactorial and contradictory role is the IL-6. In CD is a main pro-inflammatory cytokine, and in RE its secretion is observed in both pro and antiinflammatory situations. Also, IL-6 in low glycogen and high insulin concentrations in blood circulation is secreted and act as a regulator. These parameters present that the IL-6 interferes in many of the body's mechanisms which probably make the participation in RE training program an unstable condition for health (Fig. 1).

\section{CONCLUSIONS}

$\mathrm{RE}$ training program is proposed in mild intensities in CD patients as an anti-inflammatory factor for the repairment of intestinal tissue and for the increasement of muscle strength and lean mass. Besides this, there are mechanisms that seem to complicate the contribution of RE which interferes in both inflammatory and anti-inflammatory situations. Also, the lack of literature about the effect of RE on CD patients makes it difficult to conclude in any direction. Thus, this review discusses the possibility that RE training program may increase the risk of exacerbation in CD symptoms. Also, there are contradictory IL-6 mechanisms in both $\mathrm{CD}$ and RE. So, the use of a RE training program in $\mathrm{CD}$ patients needs more research for safer participation.

\section{ADDITIONAL INFORMATION}

\section{Funding Source}

The authors received no financial support for the research, authorship, and/or publication of this article.

\section{Conflict of Interest}

No potential conflict of interest relevant to this article was reported.

\section{Author Contribution}

Writing and approval of final manuscript: Papadimitriou K.

\section{ORCID}

Papadimitriou K http://orcid.org/0000-0002-7415-3582

\section{REFERENCES}

1. Engels M, Cross RK, Long MD. Exercise in patients with inflammatory bowel diseases: current perspectives. Clin Exp Gastroenterol 2017;11:1-11.

2. Legeret C, Mählmann L, Gerber M, et al. Favorable impact of long-term exercise on disease symptoms in pediatric patients with inflammatory bowel disease. BMC Pediatr 2019;19:297.

3. Holik D, Včev A, Milostić-Srb A, et al. The effect of daily physical activity on the activity of inflammatory bowel diseases in therapy-free patients. Acta Clin Croat 2019;58:202-212.

4. Mazur-Bialy AI, Bilski J, Wojcik D, et al. Beneficial effect of voluntary exercise on experimental colitis in mice fed a high-fat diet: the role of irisin, adiponectin and proinflammatory biomarkers. Nutrients 2017;9:410.

5. Hashash JG, Binion DG. Exercise and inflammatory bowel disease: insights into etiopathogenesis and modification of clinical course. Gastroenterol Clin North Am 2017;46:895905.

6. DeFilippis EM, Tabani S, Warren RU, Christos PJ, Bosworth $\mathrm{BP}$, Scherl EJ. Exercise and self-reported limitations in patients with inflammatory bowel disease. Dig Dis Sci 2016;61:215220.

7. Melinder C, Hiyoshi A, Hussein O, Halfvarson J, Ekbom A, Montgomery S. Physical fitness in adolescence and subsequent inflammatory bowel disease risk. Clin Transl Gastroenterol 2015;6:e121.

8. Tew GA, Jones K, Mikocka-Walus A. Physical activity habits, limitations, and predictors in people with inflammatory bowel disease: a large cross-sectional online survey. Inflamm 
Bowel Dis 2016;22:2933-2942.

9. Werkstetter KJ, Ullrich J, Schatz SB, Prell C, Koletzko B, Koletzko S. Lean body mass, physical activity and quality of life in paediatric patients with inflammatory bowel disease and in healthy controls. J Crohns Colitis 2012;6:665-673.

10. Chen Y, Noble EG. Is exercise beneficial to the inflammatory bowel diseases? An implication of heat shock proteins. Med Hypotheses 2009;72:84-86.

11. Lee SH, Kwon JE, Cho ML. Immunological pathogenesis of inflammatory bowel disease. Intest Res 2018;16:26-42.

12. Marchioni Beery RM, Li E, Fishman LN. Impact of pediatric inflammatory bowel disease diagnosis on exercise and sports participation: patient and parent perspectives. World J Gastroenterol 2019;25:4493-4501.

13. Bilski J, Brzozowski B, Mazur-Bialy A, Sliwowski Z, Brzozowski T. The role of physical exercise in inflammatory bowel disease. Biomed Res Int 2014;2014:429031.

14. Bilski J, Mazur-Bialy AI, Brzozowski B, et al. Moderate exercise training attenuates the severity of experimental rodent colitis: the importance of crosstalk between adipose tissue and skeletal muscles. Mediat Inflamm 2015;2015:605071.

15. Greenley RN, Naftaly JP, Walker RJ, Kappelman MD, Martin CF, Schneider KL. Sports participation in youth with inflammatory bowel diseases: the role of disease activity and subjective physical health symptoms. Inflamm Bowel Dis 2018;24: 247-253.

16. Stokes T, Hector AJ, Morton RW, McGlory C, Phillips SM. Recent perspectives regarding the role of dietary protein for the promotion of muscle hypertrophy with resistance exercise training. Nutrients 2018;10:180.

17. Schoenfeld BJ. The mechanisms of muscle hypertrophy and their application to resistance training. J Strength Cond Res 2010;24:2857-2872.

18. Elia J, Kane S. Adult inflammatory bowel disease, physical rehabilitation, and structured exercise. Inflamm Bowel Dis 2018;24:2543-2549.

19. Przybyla B, Gurley C, Harvey JF, et al. Aging alters macrophage properties in human skeletal muscle both at rest and in response to acute resistance exercise. Exp Gerontol 2006;41: 320-327.

20. Vasconcelos ES. Inflammatory response induced by resistance exercise. MOJ Immunol 2018;6:110-113.

21. Coffey VG, Hawley JA. The molecular bases of training adaptation. Sports Med 2007;37:737-763.

22. Wiroth JB, Filippi J, Schneider SM, et al. Muscle performance in patients with Crohn's disease in clinical remission. Inflamm
Bowel Dis 2005;11:296-303.

23. Cabalzar AL, Oliveira DJ, Reboredo MD, Lucca FA, Chebli JM, Malaguti C. Muscle function and quality of life in the Crohn's disease. Fisioter Mov 2017;30:337-345.

24. Robinson RJ, Krzywicki T, Almond L, et al. Effect of a low-impact exercise program on bone mineral density in Crohn's disease: a randomized controlled trial. Gastroenterology 1998;115:36-41.

25. Candow D, Rizzi A, Chillibeck P, Worobetz L. Effect of resistance training on Crohn's disease. Can J Appl Physiol 2002; 27:S7-S8.

26. Pérez CA. Prescription of physical exercise in Crohn's disease. J Crohns Colitis 2009;3:225-231.

27. De Souza Tajiri GJ, de Castro CL, Zaltman C. Progressive resistance training improves muscle strength in women with inflammatory bowel disease and quadriceps weakness. J Crohns Colitis 2014;8:1749-1750.

28. Cronin O, Barton W, Moran C, et al. Moderate-intensity aerobic and resistance exercise is safe and favorably influences body composition in patients with quiescent inflammatory bowel disease: a randomized controlled cross-over trial. BMC Gastroenterol 2019;19:29.

29. Pourrahim Ghouroghchi A, Pahlevani M. Effects of maximal and sub-maximal resistance exercise on muscle damage, inflammation, intrinsic antioxidant in non-athlete men. Koomesh 2020;22:351-358.

30. Abraham C, Cho JH. Inflammatory bowel disease. N Engl J Med 2009;361:2066-2078.

31. Lee JH, Jun HS. Role of myokines in regulating skeletal muscle mass and function. Front Physiol 2019;10:42.

32. Carrillo JLM, Del Campo JOM, Coronado OG, Gutiérrez PTV, Cordero JFC, Juárez JV. Adipose tissue and inflammation. In: Szablewski L, ed. Adipose tissue. London: IntechOpen, 2018. https://doi.org/10.5772/intechopen.74227.

33. Raines C, Frosig T, Escobar KA, Cotter JA, Schick EE. Acute resistance exercise at varying volume loads does not enhance plasma interleukin-6. Int J Kinesiol Sports Sci 2020;8:37-42.

34. Hirose L, Nosaka K, Newton M, et al. Changes in inflammatory mediators following eccentric exercise of the elbow flexors. Exerc Immunol Rev 2004;10:75-90.

35. Neurath MF, Finotto S. IL-6 signaling in autoimmunity, chronic inflammation and inflammation-associated cancer. Cytokine Growth Factor Rev 2011;22:83-89.

36. Atreya R, Neurath MF. Involvement of IL-6 in the pathogenesis of inflammatory bowel disease and colon cancer. Clin Rev Allergy Immunol 2005;28:187-196. 
37. Subramaniam K, Fallon K, Ruut T, et al. Infliximab reverses inflammatory muscle wasting (sarcopenia) in Crohn's disease. Aliment Pharmacol Ther 2015;41:419-428.

38. Barbieri M, Ferrucci L, Ragno E, et al. Chronic inflammation and the effect of IGF-I on muscle strength and power in older persons. Am J Physiol Endocrinol Metab 2003;284:E481E487.

39. Valentini L, Schaper L, Buning C, et al. Malnutrition and impaired muscle strength in patients with Crohn's disease and ulcerative colitis in remission. Nutrition 2008;24:694-702. 